\title{
A national survey on the implementation of key infection prevention and control structures in German hospitals: results from 736 hospitals conducting the WHO Infection Prevention and Control Assessment Framework (IPCAF)
}

Seven Johannes Sam Aghdassi ${ }^{1,2^{*}}$ (D) Sonja Hansen ${ }^{1,2}$, Peter Bischoff ${ }^{1,2}$, Michael Behnke ${ }^{1,2}$ and Petra Gastmeier ${ }^{1,2}$

\begin{abstract}
Background: Healthcare-associated infections (HAl) pose a burden on healthcare providers worldwide. To prevent HAI and strengthen infection prevention and control (IPC) structures, the WHO has developed a variety of tools and guidelines. Recently, the WHO released the Infection Prevention and Control Assessment Framework (IPCAF), a questionnaire-like tool designed for assessing IPC structures at the facility level. The IPCAF reflects the eight WHO core components of IPC. Data on the implementation of IPC measures in German hospitals are scarce. Therefore, it was our objective to utilize the IPCAF in order to gather information on the current state of IPC implementation in German hospitals, as well as to promote the IPCAF to a broad audience.

Methods: The National Reference Center for Surveillance of Nosocomial Infections (NRZ) sent a translated version of the IPCAF to 1472 acute care hospitals in Germany. Data entry and transfer to the NRZ was done electronically between October and December 2018. The IPCAF was conceived in a way that depending on the selected answers a score was calculated, with 0 being the lowest possible and 800 the highest possible score. Depending on the overall score, the IPCAF allocated hospitals to four different "IPC levels": inadequate, basic, intermediate, and advanced.

Results: A total of 736 hospitals provided a complete dataset and were included in the data analysis. The overall median score of all hospitals was 690, which corresponded to an advanced level of IPC. Only three hospitals (0.4\%) fell into the category "basic", with 111 hospitals (15.1\%) being "intermediate" and 622 hospitals (84.5\%) being "advanced". In no case was the category "inadequate" allocated. More profound differences were found between the respective core components. Components on multimodal strategies and workload, staffing, ward design and bed occupancy revealed the lowest scores.

\footnotetext{
* Correspondence: seven-johannes-sam.aghdassi@charite.de

${ }^{1}$ Charité - Universitätsmedizin Berlin, corporate member of Freie Universität Berlin, Humboldt-Universität zu Berlin, and Berlin Institute of Health, Institute of Hygiene and Environmental Medicine, Berlin, Germany

${ }^{2}$ National Reference Center for Surveillance of Nosocomial Infections, Berlin, Germany
} 
(Continued from previous page)

Conclusions: IPC key aspects in general are well established in Germany. Potentials for improvement were identified particularly with regard to workload and staffing. Insufficient implementation of multimodal strategies was found to be another relevant deficit. Our survey represents a successful attempt at promoting the IPCAF and encouraging hospitals to utilize WHO tools for self-assessment.

Keywords: Infection prevention, Implementation, Survey, Surveillance, Healthcare-associated infection

\section{Background}

Healthcare-associated infections (HAI) pose one of the most severe threats to the health of patients and remain a challenge for healthcare providers worldwide [1]. A recent point prevalence survey conducted in 28 EU-countries and Serbia revealed an estimated prevalence of patients with HAI in acute care hospitals of $6.5 \%$ [2]. When extrapolating prevalence data to estimate the burden of HAI on the healthcare system, it is estimated that over 2.6 million HAI occur annually in the EU. Further extrapolations suggest that these HAI account for a total of 501 disability-adjusted life years (DALYs) per 100,000 general population and an attributable number of over 90,000 deaths per year [3]. Although these figures solely apply to the European context, various studies have illustrated that HAI are also a problem in healthcare settings outside the EU, particularly in low- and middle-income countries [4]. Accordingly, institutions such as the World Health Organization (WHO) and others have in the past placed a high emphasis on developing and promoting strategies to prevent HAI [5-7].

As one of their key documents to strengthen infection prevention and control (IPC) aspects, the WHO has released the "Guidelines on Core Components of Infection Prevention and Control Programmes" offering countries as well as individual healthcare facilities an orientation on how to establish and strengthen IPC activities [8]. At the facility level, the WHO distinguishes between eight core components (CC), which address different aspects of IPC. These are:

- IPC program (CC1)

- IPC guidelines (CC2)

- IPC education (CC3)

- HAI surveillance (CC4)

- Multimodal strategies (CC5)

- Monitoring/audit of IPC practices and feedback (CC6)

- Workload, staffing and bed occupancy (CC7)

- Environments, materials and equipment for IPC (CC8)

To facilitate the establishment of IPC structures, the WHO has released manuals giving advice on how to implement the WHO Guidelines on Core Components of Infection Prevention and Control Programmes at a national and at a facility level [9]. Especially at the facility level, implementation of IPC key aspects differs widely, not only between countries of different income levels, but also within countries themselves [10-13]. Therefore, to provide healthcare facilities with an additional tool to assess, analyze and improve IPC activities at their facilities, the WHO has recently released the Infection Prevention and Control Assessment Framework (IPCAF) [14]. In form of a questionnaire, facilities can answer questions relating to IPC with the objective to determine strengths and weaknesses.

Previous studies have investigated the applicability and feasibility of other WHO tools, such as the WHO Hand Hygiene Self-Assessment Framework and the multimodal approach at hand hygiene in general [15-18], as well as the Water and sanitation for health facility improvement tool (WASH FIT) [19]. Studies have repeatedly demonstrated the feasibility and reliability of these tools and approaches. Due to the recent release of the IPCAF, the tool has not yet been applied on an equally broad range as the tools mentioned above.

In 2011, the German Protection against Infection Act ("Infektionsschutzgesetz") was revised, augmenting the importance of IPC in hospitals. However, data on the implementation of IPC measures and structures in German hospitals are scarce. Thus, it was our objective to describe the current state of implementation of key IPC aspects, as defined by the WHO core components, in German hospitals using the IPCAF. Additionally, we wanted to promote the IPCAF to motivate hospitals in Germany to perform an IPC self-assessment.

\section{Methods}

In Germany, HAI surveillance is well established. Over 2000 hospitals have participated (i.e. at least temporarily provided data) in the German nosocomial infection surveillance system "KISS" (Krankenhaus-Infektions-Surveillance-System) since its establishment in the 1990s. Annually, surveys are sent to the participating hospitals to address topics of current interest, which are relevant within the context of surveillance and HAI prevention in Germany.

The National Reference Centre for Surveillance of Nosocomial Infections (NRZ) in Germany translated the 
IPCAF into German. On the first of October 2018 the translated version was sent to 1472 acute care hospitals in Germany as the annual KISS-survey in the form of a link to a survey webpage, into which all data were entered. Data entry was possible until the end of December 2018. Table e1 to be found in the Additional file 1 illustrates structural characteristics of these 1472 hospitals. Participation was on a voluntary basis. After completing the survey, the results were automatically transferred to the NRZ. The received data were not linked to surveillance data or other data such as alcoholic hand rub consumption of the participating hospitals due to privacy and data protection regulations. The translated version of the IPCAF can be found in the Additional file 2 of this article.

As mentioned above, the IPCAF was conceived in a way that individual questions on IPC aspects had to be answered. Every possible answer of a question was allocated a score. Following the concept of the eight core components of IPC, the IPCAF was divided into eight sections. For every core component the scores of the individual questions were aggregated. A maximum score per core component of 100 was possible. The final IPCAF score was calculated by adding the scores of all eight core components (i.e. maximum total score possible was 800 ). Depending on the final score, the hospitals were grouped into four different IPC categories:

- 0-200 points: inadequate

- 201-400 points: basic

- 401-600 points: intermediate

- 601-800 points: advanced
After reception of the data, the NRZ conducted a descriptive analysis for the total IPCAF score, as well as for the scores of the respective core components and for some selected individual questions of particular interest.

All data were anonymized and collected in accordance with paragraph 23 of the German federal law, German Protection against Infection Act ("Infektionsschutzgesetz"), which regulates the prevention and control of infectious diseases in humans. Therefore, ethical approval and informed consent were not required.

\section{Results}

Altogether, 739 hospitals (response rate of 50.2\%) conducted the IPCAF and transferred data to the NRZ. Of these datasets, three were incomplete and therefore excluded from all analyses. As a result, data from a total of 736 hospitals were included and further analyzed. The overall median score, which was attributed to the participating hospitals, was 690 , with an interquartile range between 640 and 730 . When grouped by score into the above-mentioned IPC categories, only three hospitals $(0.4 \%)$ fell into the category "basic", with 111 hospitals (15.1\%) being "intermediate" and 622 hospitals $(84.5 \%)$ being "advanced". In no case was the category "inadequate" (less than 201 points) allocated. Figure 1 illustrates the distribution of scores among participating hospitals.

Differences were found with regard to the score of the individual components. Table 1 illustrates the mean, median, first and third quartile, as well as the tenth and 90th percentile for the overall IPCAF score and for the scores of each individual CC. CC7 with its focus on workload, staffing, ward design and bed occupancy had

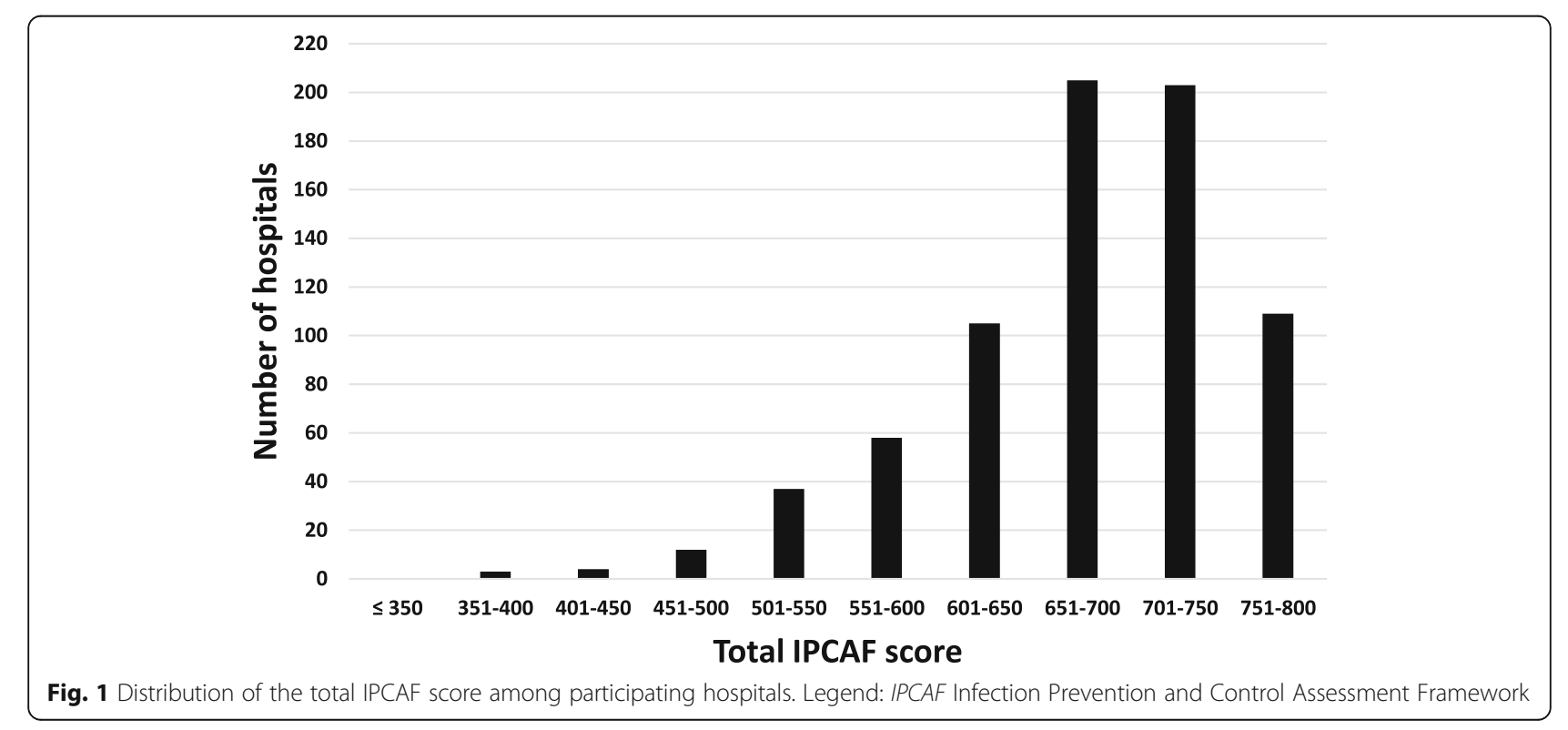


Table 1 Distribution of results of the total IPCAF score and scores per core component

\begin{tabular}{lllllll}
\hline Component & \multicolumn{2}{l}{ Score } & & & & \\
\cline { 2 - 7 } & Q10 & Q25 & Q50 & Q75 & Q90 & Mean \\
\hline CC1 & 67.5 & 80 & 88.8 & 95 & 100 & 85.7 \\
CC2 & 87.5 & 94.4 & 100 & 100 & 100 & 95.7 \\
CC3 & 65 & 75 & 85 & 90 & 100 & 82.7 \\
CC4 & 72.5 & 85 & 92.5 & 97.5 & 100 & 88.9 \\
CC5 & 40 & 60 & 80 & 90 & 95 & 71.3 \\
CC6 & 62.5 & 75 & 85 & 92.5 & 97.5 & 82.7 \\
CC7 & 45 & 60 & 75 & 90 & 95 & 74.1 \\
CC8 & 90 & 95 & 97.5 & 100 & 100 & 96.1 \\
Total & 575 & 640 & 690 & 730 & 762.5 & 677.3 \\
\hline
\end{tabular}

IPCAF Infection Prevention and Control Assessment Framework, CC core component, Q10 tenth percentile, Q25 first quartile, Q50 median, Q75 third quartile, Q90 90th percentile

the lowest median score (75), while CC5 (multimodal strategies) had the lowest mean score (71). Guidelines (CC2) and environment/infrastructure (CC8) were the two components with the highest scores. The median score of CC2 was 100 and the mean score of CC8 was 96. The range of scores per component, defined as the range between the tenth and the 90th percentile was broadest for the component on multimodal strategies (CC5) and narrowest for environment/infrastructure (CC8).

A deeper examination of the IPCAF dataset into the level of individual questions and answering patterns yielded diverse results. Due to the complexity of the IPCAF, we cannot illustrate all of these results in this article, but will instead focus on selected topics of particular interest and with a relatively large variability between hospitals. For the purpose of conciseness, we will primarily look at the components with the lowest scores, which were multimodal strategies (CC5) and workload/staffing (CC7). The majority of hospitals (645; $88 \%)$ reported that multimodal strategies were utilized to implement IPC interventions. However, only 541 hospitals $(74 \%)$ stated that an interdisciplinary team was involved in implementing these interventions and 545 hospitals (74\%) reported involving staff designated for quality and patient-safety improvement in order to conceive and promote multimodal strategies. Bundles and checklists were found to be rather prevalent, with 625 hospitals (85\%) using them as an integral part of their multimodal strategies. Further results of $\mathrm{CC} 5$ per multimodal strategy are shown in Table 2 .

In CC7, staffing, ward design and bed occupancy aspects were addressed. Two thirds of the participating hospitals (490) reported conducting an assessment of staffing needs at the facility using national or international standards. Correspondingly, 255 hospitals (35\%) stated that they did not have a system in place to react to results of a staffing needs assessment, and only 234 hospitals (32\%) stated that they maintained a defined healthcare worker to patient ratio at all times in the entire facility. Additional results of $\mathrm{CC} 7$ on ward design and bed occupancy are illustrated in Table 3, along with other questions of particular relevance and interest from other core components.

A full description of all questions and the answers we received from the participating hospitals can be found in the Additional file 3 of this article.

\section{Discussion}

To our best knowledge, our survey represents one of the first applications of the IPCAF on a broad scale. By making use of the existing surveillance structures in Germany, we were able to distribute a translated version of the IPCAF to a large number of recipients and thereby generate a great amount of data. The primary conclusion we can draw from the data received, is that in general IPC structures and activities are well established in Germany. Collectively, the participating hospitals reached a median score of 690 , which by the definitions applied in the IPCAF, translated to an advanced IPC level. However, with some hospitals falling into the categories basic or intermediate, our survey demonstrated a certain degree of heterogeneity and a potential for improvement. With regard to the individual components of the IPCAF, we found substantial differences between the respective scores. The first core component focuses on the existence and characteristics of an IPC program. The median score of 89 revealed that IPC programs are generally widely established in Germany. This could be interpreted as a result of the reform of the German Protection against Infection Act, which increased the awareness of IPC aspects in the country. Nevertheless, when focusing on specific questions of $\mathrm{CC} 1$, more diverse results were seen. For instance, around $40 \%$ of hospitals stated that their IPC program lacked clearly defined objectives and less than $40 \%$ of hospitals reported having defined future targets for their IPC program. Along with the non-optimal results concerning IPC staffing and the lack of support from the senior facility leadership in a substantial proportion of hospitals (Table 3), these results illustrate a remarkable potential for improvement.

Scores for IPC guidelines (CC2) and IPC training and education (CC3) were generally high with few exceptions. Potential for improvement, however, can be found with reference to the methods applied to perform IPC training. Only around 40\% of hospitals reported utilizing interactive teaching methods (e.g. bedside training), which have been proven in the past to be an effective form of education [20, 21]. However, this finding 
Table 2 Results per multimodal strategy from IPCAF core component 5

\begin{tabular}{|c|c|c|}
\hline Element & Answer & Number (\%) \\
\hline \multirow[t]{3}{*}{ System change } & Element not included in multimodal strategies & $111(15.1)$ \\
\hline & $\begin{array}{l}\text { Interventions to ensure the necessary infrastructure and continuous availability of supplies } \\
\text { are in place }\end{array}$ & $218(29.6)$ \\
\hline & $\begin{array}{l}\text { Interventions to ensure the necessary infrastructure and continuous availability of supplies } \\
\text { are in place and addressing ergonomics and accessibility, such as the best placement of } \\
\text { central venous catheter set and tray }\end{array}$ & $407(55.3)$ \\
\hline \multirow[t]{3}{*}{ Education and training } & Element not included in multimodal strategies & $32(4.3)$ \\
\hline & Written information and/or oral instruction and/or e-learning only & $439(59.6)$ \\
\hline & Additional interactive training sessions (includes simulation and/or bedside training) & $265(36.0)$ \\
\hline \multirow[t]{3}{*}{ Monitoring and feedback } & Element not included in multimodal strategies & $83(11.3)$ \\
\hline & $\begin{array}{l}\text { Monitoring compliance with process or outcome indicators (for example, audits of } \\
\text { hand hygiene or catheter practices) }\end{array}$ & $183(24.9)$ \\
\hline & $\begin{array}{l}\text { Monitoring compliance and providing timely feedback of monitoring results to } \\
\text { health care workers and key players }\end{array}$ & $470(63.9)$ \\
\hline \multirow[t]{3}{*}{ Communications and reminders } & Element not included in multimodal strategies & $124(16.8)$ \\
\hline & Reminders, posters, or other advocacy/awareness-raising tools to promote the intervention & $385(52.3)$ \\
\hline & $\begin{array}{l}\text { Additional methods/initiatives to improve team communication across units and } \\
\text { disciplines (for example, by establishing regular case conferences and feedback rounds) }\end{array}$ & $227(30.8)$ \\
\hline \multirow[t]{3}{*}{ Safety climate and culture change } & Element not included in multimodal strategies & $257(34.9)$ \\
\hline & $\begin{array}{l}\text { Managers/leaders show visible support and act as champions and role models, promoting } \\
\text { an adaptive approach and strengthening a culture that supports IPC, patient safety and quality }\end{array}$ & $321(43.6)$ \\
\hline & $\begin{array}{l}\text { Additionally, teams and individuals are empowered so that they perceive ownership of } \\
\text { the intervention (for example, by participatory feedback rounds) }\end{array}$ & $158(21.5)$ \\
\hline
\end{tabular}

IPCAF Infection Prevention and Control Assessment Framework

corresponds with data from other fields of medicine, which see a decline in the application of this didactic method [22, 23]. Moreover, IPC education could also be improved by implementing IPC aspects into the training of other specialties of medicine, which currently less than $40 \%$ of hospitals seem to undertake systematically (Table 3 ).

Multimodal strategies, which are the main topic of the fifth core component, are a relatively new concept in the practice of infection control $[15,24,25]$. We saw a mean score of only 71 in this component, which illustrated a clear deficit. CC5 yielded the most diverse results of all components, indicating that this rather novel approach at IPC is already implemented in a considerable number of German hospitals, and yet, still represents a relevant potential for improvement. This was especially true for questions on the individual elements of multimodal strategies, such as system change, education and training, etc. (Table 2).

HAI surveillance (CC4), as well as monitoring and auditing of IPC processes (CC6), were revealed to be well established in German hospitals. As demonstrated in many publications, Germany has a well-functioning surveillance network with a long history [26]. Timely and appropriate feedback of surveillance data is one of the key aspects of conducting successful surveillance
[27]. Our survey, however, suggested a deficit concerning the way that surveillance data is fed back in many German hospitals. A third of participating hospitals reported giving no feedback at all or in written/oral form only, not embracing a more interactive approach (Table 3).

With the eighth core component investigating structures such as water and electricity supply, it is obvious that this component is more geared towards low- or middle-income settings, thus not being fully applicable to the German context. Unsurprisingly, scores for this component were generally very high.

Understaffing has previously been demonstrated to be a risk for the occurrence of HAI $[28,29]$. Therefore, the deficits found to exist with regard to CC7, which focuses, among other aspects, on the healthcare worker to patient ratio as a key aspect of IPC, gain relevance. Less than a third of all hospitals recorded maintaining a defined healthcare worker to patient ratio in the entire facility at all times. Remarkably, around a third of all hospitals, reportedly, were not conducting a staffing needs assessment using national or international standards and had no system in place to react to a change in the demand for staff. These findings confirm the previously described shortage of qualified staff for patient care in German hospitals [30] and represents one of the 
Table 3 Selected results of the IPCAF from various core components

\begin{tabular}{|c|c|c|}
\hline Topic & Answer & Number (\%) \\
\hline \multirow[t]{3}{*}{ Existence of IPC program (CC1) } & Not existent & $32(4.3)$ \\
\hline & Existent but no clearly defined objectives & $262(35.6)$ \\
\hline & Existent with clearly defined objectives and annual activity plan & $442(60.1)$ \\
\hline \multirow[t]{4}{*}{ Defined IPC objectives in critical areas (CC1) } & No IPC objectives & $43(5.8)$ \\
\hline & IPC objectives only & $137(18.6)$ \\
\hline & IPC objectives and measurable outcome indicators & $285(38.7)$ \\
\hline & IPC objectives and measurable outcome indicators and future targets & $271(36.8)$ \\
\hline \multirow[t]{2}{*}{ Senior facility leadership (CC1) } & Does not provide specific allocated budget & $230(31.3)$ \\
\hline & Provides specific allocated budget & $506(68.8)$ \\
\hline \multirow[t]{2}{*}{ Senior facility leadership (CC1) } & Does not show demonstrable support & $213(28.9)$ \\
\hline & Shows demonstrable support & $523(71.1)$ \\
\hline \multirow[t]{3}{*}{ IPC training of healthcare-workers (CC3) } & Not existent & $1(0.1)$ \\
\hline & Only in written and/or oral and/or online form & $437(59.4)$ \\
\hline & Interactive training (e.g. bedside teaching) & $298(40.5)$ \\
\hline \multirow[t]{3}{*}{ IPC training and training of other specialties (CC3) } & IPC aspects not integrated into training of other specialties & $146(19.8)$ \\
\hline & IPC aspects integrated into training of some other specialties & $302(41.0)$ \\
\hline & IPC aspects integrated into training of all other specialties & $288(39.1)$ \\
\hline \multirow[t]{3}{*}{ Feedback of surveillance data (CC4) } & No annual feedback & $8(1.1)$ \\
\hline & Annual feedback in written and/or oral form only & $237(32.2)$ \\
\hline & Annual feedback via presentation and interactive problem-solution finding & $491(66.7)$ \\
\hline \multirow[t]{3}{*}{ Ward design (CC7) } & Not in accordance with international standards & $90(12.2)$ \\
\hline & Certain departments in accordance with international standards & $199(27.0)$ \\
\hline & All departments in accordance with international standards & $447(60.7)$ \\
\hline \multirow[t]{3}{*}{ Patient placement in corridor beds outside the room (CC7) } & More frequently than twice a week & $61(8.3)$ \\
\hline & Less frequently than twice a week & $162(22.0)$ \\
\hline & Never & $513(69.7)$ \\
\hline
\end{tabular}

IPCAF Infection Prevention and Control Assessment Framework, IPC infection prevention and control, CC core component

most relevant findings of our survey. Along with the deficits for ward design and bed occupancy, illustrated in Table 3, we can conclude that improvements in the field of workload and staffing are of urgent need in German hospitals.

When interpreting the data generated through this survey several limitations have to be recognized. Among the most relevant were:

- In spite of numerous footnotes and explanations provided, the IPCAF required a profound understanding of the WHO terminology and underlying concepts. Relatively new concepts, such as multimodal strategies, were not understood by every respondent, leaving room for misinterpretation and false reporting.

- The IPCAF collected information, which, although handled discreetly by the NRZ, may have been perceived as potentially compromising by some hospitals. Therefore, in some cases questions could have been answered wrongly purposefully to achieve a higher score.

- Facilities with a high interest in aspects of IPC may have had a greater interest in completing the survey (overall response rate of 50.2\%) and may therefore be overrepresented.

- Due to the electronic form of data entry and anonymization of data, participants were not able to retroactively correct data entry mistakes once the survey was completed and data sent to the NRZ. This may explain some unexpected individual results.

- The IPCAF did not collect information such as hospital size, type or ownership, which would be helpful to better interpret some of the data generated.

Besides these limitations, our survey had numerous strengths. The most relevant were:

- A high number of hospitals participated, which allowed for careful extrapolations to the national level. 
- Germany has a long history of surveillance, which increased the understanding of many concepts addressed by the IPCAF and the readiness of hospitals to participate in the survey.

- The translation of the survey into German allowed participants to answer the IPCAF in their native language, thereby reducing the language barrier.

- The NRZ provided help and advice for hospitals that had difficulties interpreting certain questions.

\section{Conclusion}

IPC structures and processes are in general well established in Germany. In particular, this can be concluded for IPC guidelines and HAI surveillance. Conversely, a potential for improvement was discovered especially with regard to the implementation of multimodal strategies and for aspects of workload and appropriate healthcare worker staffing. To our best knowledge, our survey represents the first broad application of the IPCAF, and will serve as a useful orientation for future applications within Germany and in other countries. Developments and trends may become apparent through repeated application of the IPCAF. The primary purpose of the IPCAF was to enable healthcare facilities to perform an IPC self-assessment. Lessons learned from the IPCAF by the participating hospitals (e.g. through identifying deficits) may reveal themselves in years to come in form of activities to strengthen IPC structures.

\section{Additional files}

Additional file 1: Structural characteristics of 1472 German acute care hospitals invited to participate in the WHO Infection Prevention and control assessment framework (IPCAF). (DOCX $12 \mathrm{~kb}$ )

Additional file 2: Infection Prevention and control assessment framework. (IPCAF) (German translation). (DOCX $82 \mathrm{~kb}$ )

Additional file 3: Results of the Infection Prevention and Control Assessment Framework (IPCAF) in 736 German hospitals. (DOCX 45 kb)

\section{Abbreviations}

CC: Core component; DALYs: Disability-adjusted life years; HAl: Healthcareassociated infection(s); IPC: Infection prevention and control; IPCAF: Infection Prevention and Control Assessment Framework; KISS: Krankenhaus-InfektionsSurveillance-System; NRZ: National Reference Center for Surveillance of Nosocomial Infections; WASH FIT: Water and sanitation for health facility improvement tool; WHO: World Health Organization

\section{Acknowledgements \\ The authors wish to thank all of the KISS participants for their efforts and work and the German Ministry of Health for supporting the National Reference Center for Surveillance of Nosocomial infections. Furthermore, the authors would like to extend many thanks to colleagues of the WHO for their continuous dedication and efforts to promote novel approaches at infection prevention and control.}

\section{Funding}

The study was not funded.

\section{Availability of data and materials}

The datasets used and analyzed in the context of this survey are available from the corresponding author upon reasonable request.

\begin{abstract}
Authors' contributions
SA, SH, PB and PG defined the objectives of the study and led the study design and development. MB provided the programming necessary for the management of the received data. SA drafted the manuscript with the help and input of all listed co-authors. All authors approved the final version of the manuscript.
\end{abstract}

\section{Ethics approval and consent to participate}

Not applicable, because all data were surveillance-based data which were obtained in accordance with the German Protection against Infection Act ("Infektionsschutzgesetz").

\section{Consent for publication}

Not applicable, because all data were surveillance-based data which were obtained in accordance with the German Protection against Infection Act ("Infektionsschutzgesetz").

\section{Competing interests}

The authors declare that they have no competing interests.

\section{Publisher's Note}

Springer Nature remains neutral with regard to jurisdictional claims in published maps and institutional affiliations.

Received: 1 March 2019 Accepted: 30 April 2019

Published online: 08 May 2019

\section{References}

1. WHO. Report on the burden of endemic health care-associated infection worldwide. World Health Organization. 2011. http://apps.who.int/iris/ bitstream/10665/80135/1/9789241501507_eng.pdf. Accessed 27 Feb 2019.

2. Suetens C, Latour K, Karki T, Ricchizzi E, Kinross P, Moro ML, et al. Prevalence of healthcare-associated infections, estimated incidence and composite antimicrobial resistance index in acute care hospitals and long-term care facilities: results from two European point prevalence surveys, 2016 to 2017. Euro Surveill. 2018;23(46)

3. Cassini A, Plachouras D, Eckmanns T, Abu Sin M, Blank HP, Ducomble T, et al. Burden of six healthcare-associated infections on European population health: estimating incidence-based disability-adjusted life years through a population prevalence-based modelling study. PLoS Med. 2016;13(10):e1002150.

4. Allegranzi B, Bagheri Nejad S, Combescure C, Graafmans W, Attar H, Donaldson $\mathrm{L}$, et al. Burden of endemic health-care-associated infection in developing countries: systematic review and meta-analysis. Lancet. 2011; 377(9761):228-41.

5. Centers for Disease Control and Prevention. Infection Control Guidelines Library https://www.cdc.gov/infectioncontrol/guidelines/index.html (2015). Accessed 27 Feb 2019.

6. Siegel JD, Rhinehart E, Jackson M, Chiarello L, Health care Infection control practices advisory C. Guideline for isolation precautions: preventing transmission of infectious agents in health care settings. Am J Infect Control. 2007;35(10 Suppl 2):S65-164.

7. Storr J, Twyman A, Zingg W, Damani N, Kilpatrick C, Reilly J, et al. Core components for effective infection prevention and control programmes: new WHO evidence-based recommendations. Antimicrob Resist Infect Control. 2017;6:6.

8. WHO. Guidelines on Core components of Infection Prevention and control Programmes at the national and acute health care facility level. World Health Organization. 2016. https://apps.who.int/iris/bitstream/handle/10665/ 251730/9789241549929-eng.pdf? sequence=1. Accessed 27 Feb 2019.

9. WHO. Interim practical manual supporting implementation of the $\mathrm{WHO}$ guidelines on Core components of Infection Prevention and control Programmes. World Health Organization. 2017. https://www.who.int/ infection-prevention/tools/core-components/cc-implementation-guideline. pdf. Accessed 27 Feb 2019.

10. Hansen S, Schwab F, Gropmann A, Behnke M, Gastmeier P, Consortium P. Hygiene und Sicherheitskultur in deutschen Krankenhäusern. Bundesgesundheitsbl Gesundheitsforsch Gesundheitsschutz. 2016;59(7):908-15. 
11. Struelens MJ, Wagner D, Bruce J, MacKenzie FM, Cookson BD, Voss A, et al. Status of infection control policies and organisation in European hospitals, 2001: the ARPAC study. Clin Microbiol Infect. 2006;12(8):729-37.

12. Dickstein Y, Nir-Paz R, Pulcini C, Cookson B, Beovic B, Tacconelli E, et al. Staffing for infectious diseases, clinical microbiology and infection control in hospitals in 2015: results of an ESCMID member survey. Clin Microbiol Infect. 2016;22(9):812 e9-e17.

13. Hansen S, Zingg W, Ahmad R, Kyratsis Y, Behnke M, Schwab F, et al. Organization of infection control in European hospitals. J Hosp Infect. 2015;91(4):338-45.

14. WHO. Infection Prevention and Control Assessment Framework. World Health Organization. 2018. https://www.who.int/infection-prevention/tools/ core-components/IPCAF-facility.pdf. Accessed 27 Feb 2019

15. Allegranzi B, Gayet-Ageron A, Damani N, Bengaly L, McLaws ML, Moro ML, et al. Global implementation of WHO's multimodal strategy for improvement of hand hygiene: a quasi-experimental study. Lancet Infect Dis. 2013;13(10):843-51.

16. Allegranzi B, Sax H, Bengaly L, Richet H, Minta DK, Chraiti MN, et al. Successful implementation of the World Health Organization hand hygiene improvement strategy in a referral hospital in Mali, Africa. Infect Control Hosp Epidemiol. 2010;31(2):133-41.

17. Allegranzi B, Conway L, Larson E, Pittet D. Status of the implementation of the World Health Organization multimodal hand hygiene strategy in United States of America health care facilities. Am J Infect Control. 2014;42(3):224-30.

18. Stewardson AJ, Allegranzi B, Perneger TV, Attar H, Pittet D. Testing the WHO hand hygiene self-assessment framework for usability and reliability. J Hosp Infect. 2013;83(1):30-5.

19. Weber N, Martinsen AL, Sani A, Assigbley EKE, Azzouz C, Hayter A, et al. Strengthening healthcare facilities through water, sanitation, and hygiene (WASH) improvements: a pilot evaluation of "WASH FIT" in Togo. Health Secur. 2018;16(S1):S54-65.

20. Burden AR, Torjman MC, Dy GE, Jaffe JD, Littman JJ, Nawar F, et al. Prevention of central venous catheter-related bloodstream infections: is it time to add simulation training to the prevention bundle? J Clin Anesth. 2012;24(7):555-60.

21. Zingg W, Cartier V, Inan C, Touveneau S, Theriault M, Gayet-Ageron A, et al. Hospital-wide multidisciplinary, multimodal intervention programme to reduce central venous catheter-associated bloodstream infection. PLoS One. 2014;9(4):e93898

22. Qureshi Z. Back to the bedside: the role of bedside teaching in the modern era. Perspect Med Educ. 2014;3(2):69-72.

23. Peters $\mathrm{M}$, Ten Cate O. Bedside teaching in medical education: a literature review. Perspect Med Educ. 2014:3(2):76-88.

24. Kritsotakis El, Astrinaki E, Messaritaki A, Gikas A. Implementation of multimodal infection control and hand hygiene strategies in acute-care hospitals in Greece: a cross-sectional benchmarking survey. Am J Infect Control. 2018:46(10):1097-103.

25. Oliveira AC, Gama CS, Paula AO. Multimodal strategy to improve the adherence to hand hygiene and self-assessment of the institution for the promotion and practice of hand hygiene. J Public Health (Oxf). 2018;40(1):163-8.

26. Schroder C, Schwab F, Behnke M, Breier AC, Maechler F, Piening B, et al. Epidemiology of healthcare associated infections in Germany: nearly 20 years of surveillance. Int J Med Microbiol. 2015;305(7):799-806.

27. Gastmeier P, Sohr D, Schwab F, Behnke M, Zuschneid I, Brandt C, et al. Ten years of KISS: the most important requirements for success. J Hosp Infect. 2008;70(Suppl 1):11-6.

28. Schwab F, Meyer E, Geffers C, Gastmeier P. Understaffing, overcrowding inappropriate nurse:ventilated patient ratio and nosocomial infections: which parameter is the best reflection of deficits? J Hosp Infect. 2012;80(2):133-9.

29. Fridkin SK, Pear SM, Williamson TH, Galgiani JN, Jarvis WR. The role of understaffing in central venous catheter-associated bloodstream infections. Infect Control Hosp Epidemiol. 1996;17(3):150-8.

30. Schermuly CC, Draheim M, Glasberg R, Stantchev V, Tamm G, Hartmann M, et al. Human resource crises in German hospitals--an explorative study. Hum Resour Health. 2015;13:40.

Ready to submit your research? Choose BMC and benefit from:

- fast, convenient online submission

- thorough peer review by experienced researchers in your field

- rapid publication on acceptance

- support for research data, including large and complex data types

- gold Open Access which fosters wider collaboration and increased citations

- maximum visibility for your research: over $100 \mathrm{M}$ website views per year

At BMC, research is always in progress.

Learn more biomedcentral.com/submissions 